\title{
HUA ENHANCER1 Mediates Ovule Development
}

\section{OPEN ACCESS}

Edited by:

Yuling Jiao,

Institute of Genetics and Developmental Biology, Chinese Academy of Sciences, China

Reviewed by:

Wen-hui Lin,

Shanghai Jiao Tong University, China Gabriela Carolina Pagnussat, National University of Mar del Plata, Argentina

${ }^{*}$ Correspondence: Yan Zhang

yzhang@sdau.edu.cn Sha Li

shali@sdau.edu.cn

†These authors have contributed equally to this work

Specialty section:

This article was submitted to Plant Development and EvoDevo, a section of the journal Frontiers in Plant Science

Received: 07 February 2020 Accepted: 19 March 2020

Published: 15 April 2020

Citation:

Wei S-J, Chai S, Zhu R-M, Duan C-Y, Zhang Y and Li S (2020) HUA ENHANCER1 Mediates Ovule Development.

Front. Plant Sci. 11:397. doi: $10.3389 /$ fpls.2020.00397

\author{
Shuai-Jie Weit, Sen Chait, Rui-Min Zhu, Cun-Ying Duan, Yan Zhang* and Sha Li* \\ State Key Laboratory of Crop Biology, College of Life Sciences, Shandong Agricultural University, Tai'an, China
}

Ovules are female reproductive organs of angiosperms, containing sporophytic integuments and gametophytic embryo sacs. After fertilization, embryo sacs develop into embryos and endosperm whereas integuments into seed coat. Ovule development is regulated by transcription factors (TF) whose expression is often controlled by microRNAs. Mutations of Arabidopsis DICER-LIKE 1 (DCL1), a microRNA processing protein, caused defective ovule development and reduced female fertility. However, it was not clear whether other microRNA processing proteins participate in this process and how defective ovule development influenced female fertility. We report that mutations of HUA ENHANCER1 (HEN1) and HYPONASTIC LEAVES 1 (HYL1) interfered with integument growth. The sporophytic defect caused abnormal embryo sac development and inability of mutant ovules to attract pollen tubes, leading to reduced female fertility. We show that the role of HEN1 in integument growth is cell-autonomous. Although AUXIN RESPONSE FACTOR 6 (ARF6) and ARF8 were ectopically expressed in mutant ovules, consistent with the reduction of microRNA167 in hen1, introducing arf6;arf8 did not suppress ovule defects of hen1, suggesting the involvement of more microRNAs in this process. Results presented indicate that the microRNA processing machinery is critical for ovule development and seed production through multiple microRNAs and their targets.

Keywords: integument, fertility, microRNA, HYL1, female gametophytes

\section{INTRODUCTION}

Ovule development is critical for seed yield and plant reproduction. A mature ovule is composed of sporophytic cells, i.e., integuments, and a female gametophyte (FG), called embryo sac (Schneitz et al., 1995, 1997; Christensen et al., 1997; Drews et al., 1998; Drews and Yadegari, 2002; Shi and Yang, 2011). The embryo sac forms embryo and endosperm whereas integuments develop into seed coat after fertilization. During ovule development, megasporogenesis establishes a proximal-distal polarity while megagametogenesis ensures the production of FG (Christensen et al., 1997; Drews et al., 1998; Drews and Yadegari, 2002). A critical event during megagametogenesis in Arabidopsis is the asymmetric growth of outer integuments, which finally envelops the embryo sac and leads to the anatrophy of mature ovules (Schneitz et al., 1995, 1997; Drews et al., 1998; Drews and Yadegari, 2002). Arabidopsis mutants defective in the asymmetric growth of outer integuments often shows defective formation of FG (Bencivenga et al., 2011; Chevalier et al., 2011; Wang et al., 2016), suggesting sporophytic control of gametophytic development. 
A number of transcriptional factors (TFs) regulate integument growth (Colombo et al., 2008). Mutations of INNER NO OUTER (INO), ABERRANT TESTA SHAPE (ATS), or AINTEGUMENTA (ANT) compromised the initiation and growth of integuments, resulting in a loss of the outer-inner integument organization (Elliott et al., 1996; Klucher et al., 1996; Villanueva et al., 1999; McAbee et al., 2006). Genes encoding a few homeodomain proteins, such as BELL1 (BEL1) (Reiser et al., 1995), PHABULOSA (PHB) (Sieber et al., 2004), and WUSHEL (WUS) (Gross-Hardt et al., 2002; Lieber et al., 2011), play roles in integument growth and ovule development. Except for the positive regulators of integument growth, ectopic expression of AUXIN RESPONSE FACTOR6 (ARF6) and ARF8 resulted in the arrest of integument growth (Wu et al., 2006), suggesting that there are negative regulators in this process.

Transcription factors are major targets of microRNAs (miRNAs), which are small RNAs of 20- to 24-nucleotide (nt), produced from pre-miRNA-encoding genes, and sequencespecific regulators of gene expression. Because of key roles of miRNAs in plant growth and responses to environment, their expression, processing, and turnover are tightly regulated (Dong et al., 2008; Rogers and Chen, 2013). Mutations at miRNA processing genes often result in reduced fertility (Lu and Fedoroff, 2000; Schauer et al., 2002; Olmedo-Monfil et al., 2010). Arabidopsis DICER-LIKE 1 (DCL1) is a miRNA processing protein (Kurihara and Watanabe, 2004). A mutant of DCL1, short integuments (sin1/dcl1-7), was identified from a chemical mutagenesis (Robinson-Beers et al., 1992). sin1/dcl1-7 is defective in the asymmetric growth of integuments (RobinsonBeers et al., 1992) during ovule developmental stage 3-I when a functional megaspore (FM) is formed (Schneitz et al., 1995, 1997). Efficient and precise processing of pri-miRNAs requires the interaction between DCL1 and HYPONASTIC LEAVES 1 (HYL1) (Kurihara et al., 2006), a dsRNA-binding protein (Vazquez et al., 2004; Dong et al., 2008). HUA ENHANCER1 (HEN1), a multidomain AdoMet-dependent 2'O-methyltransferase critical for miRNA biogenesis (Chen et al., 2002; Yu et al., 2005, 2010; Baranauske et al., 2015), act in the same pathway as DCL1 and HYL1 (Yang et al., 2010; Baranauske et al., 2015). Whether they participate in ovule development and how their mutations influence female fertility are not clear.

We report here that hen1-8, a hypomorphic mutant of HEN1 (Chen et al., 2002; Yu et al., 2005, 2010), and hyl1-2, a null mutant of HYL1 (Han et al., 2004; Kurihara et al., 2006; Dong et al., 2008), are defective in ovule development. Mutant ovules failed to have asymmetric integument growth, leading to abnormal embryo sac development, compromised pollen tube guidance, and thus reduced female fertility. Downregulating HEN1 specifically in outer integuments phenocopied hen1-8, suggesting a cellautonomous action. Ectopic expression of ARF6 and ARF8 and distorted auxin maximum in hen 1-8 ovules are consistent with the reduction of miRNA167, whose processing relies on HEN1 (Yu et al., 2010; Ren et al., 2012). However, introducing the arf6;arf8 double mutant did not suppress ovule defects of hen 1-8, suggesting the involvement of more microRNAs in this process.

\section{MATERIALS AND METHODS}

\section{Plant Growth and Transformation}

Arabidopsis mutants lines, including hen1-8 (Yu et al., 2010), hen1-2 (Chen et al., 2002), hyl1-2 (Vazquez et al., 2004), arf6-1 (Okushima et al., 2005), arf8-3 (Nagpal et al., 2005),

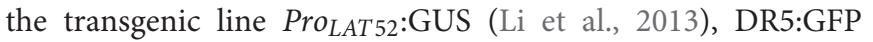
(Ulmasov et al., 1997), PIN1:GFP (Benkova et al., 2003), and Pro $_{E S 1}$ :NLS-YFP (Bencivenga et al., 2012) were described previously. Col-0 ecotype or Landsberg erecta (Ler) was used as the wild type as noted. Arabidopsis plants were grown as described (Zhou et al., 2013). In brief, the seeds were surface-sterilized and then sown on half-strength Murashige and Skoog (MS) basal medium with vitamins (Phytotechlab, SPS0519160A). Plates were placed under $4^{\circ} \mathrm{C}$ in darkness for 3 days before being moved to a growth chamber with a 16-h-light/8-h-dark cycle at $21^{\circ} \mathrm{C}$. One week later, the seedlings were transferred to a $3: 1$ mix of nutrient soil: vermiculite under same conditions with growth chamber. Plant growth, transformation, and selection were as described (Zhou et al., 2013).

\section{DNA Manipulation}

The artificial miRNA construct targeting HEN1 (amiR-HEN1) was designed with the primers ZP7781/ZP7782/ZP7783/ZP7784 using WMD3-Designer. The amiR-HEN1 was cloned into pROKII-GFP to generate Pro $_{\text {LAT 52:amiR-HEN1. Later, Pro }}$ Lat52 was replaced by ProINO to generate ProINO:amiR-HEN1. The RNAi-HEN1 fragment (2297bp to 2599bp of HEN1 coding sequence) was amplified with the primer pair ZP6753/ZP6754. The resultant PCR products were sub-cloned into the RNAi vector pTCK303 (Guo et al., 2010) to obtain the Pro $U B Q 10: R N A i-$ HEN1 construct. Later, Pro ${ }_{U B Q} 10$ was replaced by Pro $_{I N O}$ to generate Pro $_{I N O}:$ RNAi-HEN1. Pro ${ }_{H E N 1}$ was cloned into $\mathrm{pENTR/SD/D-TOPO} \mathrm{(Invitrogen)} \mathrm{with} \mathrm{the} \mathrm{primer} \mathrm{pair}$ ZP5140/ZP5173, including a 1847 bp sequence upstream of HEN1 start codon. The entry vector was used in a LR reaction with the destination vector pMD163 (Curtis and Grossniklaus, 2003) to generate Pro $_{H E N 1}$ :GUS. All primers are listed in Supplementary Table S1.

\section{Genotyping PCRs, RNA Extraction and qPCRs}

Genotyping PCRs for arf6-1 and arf8-3 were performed using following primer pairs: ZP308/ZP309 and ZP306/ZP307 for the wild copy, ZP1/ZP309 and ZP7546/ZP307 for the mutant copy of ARF6 and ARF8, respectively. Total RNAs were extracted from mature ovules using a Qiagen RNeasy plant mini kit. For qPCRs of ARF6 and ARF8 in ovules, oligo(dT)-primed cDNAs were synthesized using a FastQuant RT Kit (TIAN GEN, Cat\#KR10602). Internal controls were as described (Zhou et al., 2013). qPCRs were performed with three biological replicates. Primers used in qPCRs were ZP201/ZP202 for TUBLIN2, ZP687/ZP688 for GAPDH, ZP7207/ZP7208 for ARF6, ZP7209/ZP7210 for $A R F 8$, and ZP9325/ZP9326 for HEN1. All primers are listed in Supplementary Table S1. 


\section{RNA in situ Hybridization}

RNA in situ hybridization was performed as previously described (Zhou et al., 2013). In brief, the emasculate pistils were fixed in $4 \%$ Paraformaldehyde solution (aladdin) at $4^{\circ} \mathrm{C}$ overnight. Then the fixed tissues were embedded in Paraplast (SigmaAldrich) after dehydration and were then sectioned at $8 \mu \mathrm{m}$. RNA probes of ARF6 and ARF8 were amplified with the primer pairs ZP8093/8094 and ZP8095/8096, respectively. The sense and antisense probes were modified in vitro with digoxigeninUTP by SP6 or T7 RNA polymerases (Roche), respectively. Sections were hybridized with $1.5 \mathrm{ng} / \mu \mathrm{L}$ probes at $42^{\circ} \mathrm{C}$ overnight in a hybridization solution that contained formamide. Hybridization signals were detected by antidigoxigenin antibody (Anti-Digoxigenin-Ap Fab fragments; Roche). The samples were observed using an Olympus BX53 microscope. All primers are listed in Supplementary Table S1.

\section{Phenotype Analysis}

Pollen tube in vivo growth by histochemical GUS staining of

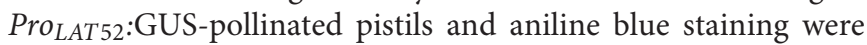
performed as described (Li et al., 2013). Whole-mount ovule clearing and CLSM of ovules were performed as described (Wang et al., 2016; Liu et al., 2019). Flowers at stage 12 were emasculated and left to grow for $12-16 \mathrm{~h}$ before pollination assays.

\section{Fluorescence Microscopy}

Lysotracker red staining was used to show cell silhouettes as described (Wang et al., 2016). CLSM of fluorescence materials was performed with a LSM880 (Zeiss) with the excitation and emission wavelengths set to $488 \mathrm{~nm} / 505-550 \mathrm{~nm}$ for YFP and GFP signals and $561 \mathrm{~nm} / 600 \mathrm{~nm}$ for RFP signals, respectively.

\section{Accession Numbers}

Arabidopsis Genome Initiative locus identifiers for the genes mentioned in this article are: AT4G20910 for HEN1; AT1G09700 for HYL1; AT3G22886 for miRNA167; AT1G30330 for ARF6; AT5G37020 for ARF8.

\section{RESULTS}

\section{hen 1-8 Shows Reduced Fertility Due to Sporophytic Female Defects}

To determine what caused the reduced fertility in hen 1-8 (Chen et al., 2002; Yu et al., 2005, 2010), we performed the following experiments. First, we observed white and wrinkled ovules dispersed among developing seeds in the maturing siliques of hen 1-8 plants, but not in those of wild type or of hen1$8 /+$ (Figures 1A,B), indicating that the reduced fertility is sporophytic. Indeed, segregation ratio by reciprocal crosses indicated that both the male and female gametophytes of hen18 were transmitted normally (Supplementary Table S2). Pollen development of hen 1-8 is also comparable to that of wild type (Supplementary Figure S1). By dissecting siliques from crosses between wild type and hen1-8, we observed reduced fertility only when hen 1-8 was used as the female parent (Figures 1A,B),

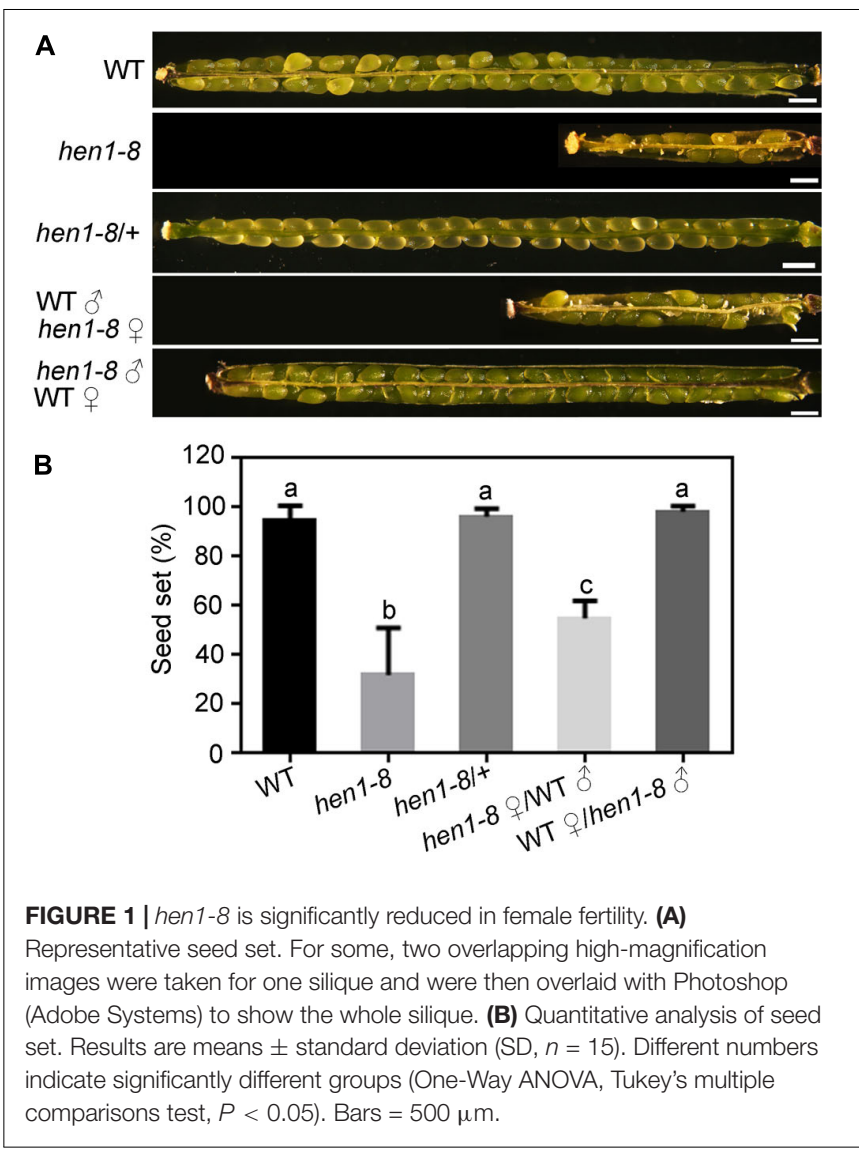

suggesting that the reduced fertility of hen 1-8 was due to sporophytic female defects.

\section{hen 1-8 Is Defective in Sporophytic Control of Ovule Development}

To determine the reason for sporophytic female defects that caused reduced fertility in hen 1-8, we examined the morphology of mature ovules by scanning electron micrographs (SEMs) and whole-mount ovule clearing (Wang et al., 2016). At maturation, wild-type ovules showed a typical anatropy with the micropyle proximal to the funiculus (Figures 2A,B). An embryo sac was clearly seen in the mature ovule of wild type (Figure 2E). By contrast, micropyle structure was not discernible in a portion of hen 1-8 ovules (Figures 2C,R). Instead, a bulge, likely a deformed embryo sac, was exposed (Figures 2D,G,H). These results suggested that ovule development is compromised in hen $1-8$.

To determine at which stage the hen 1-8 ovules started to be defective, we performed confocal laser scanning microscopy (CLSM) of developing ovules. At early stages, i.e., before the meiosis of megaspore mother cell (MMC), hen1-8 and wild type are comparable although HEN1 is expressed in ovules throughout development (Supplementary Figure S2). However, at stage 3-I when the outer integuments of wild type started rapid and asymmetric growth, extending above the inner integuments (Figure 2I), the growth of hen 1-8 outer integuments was delayed, 


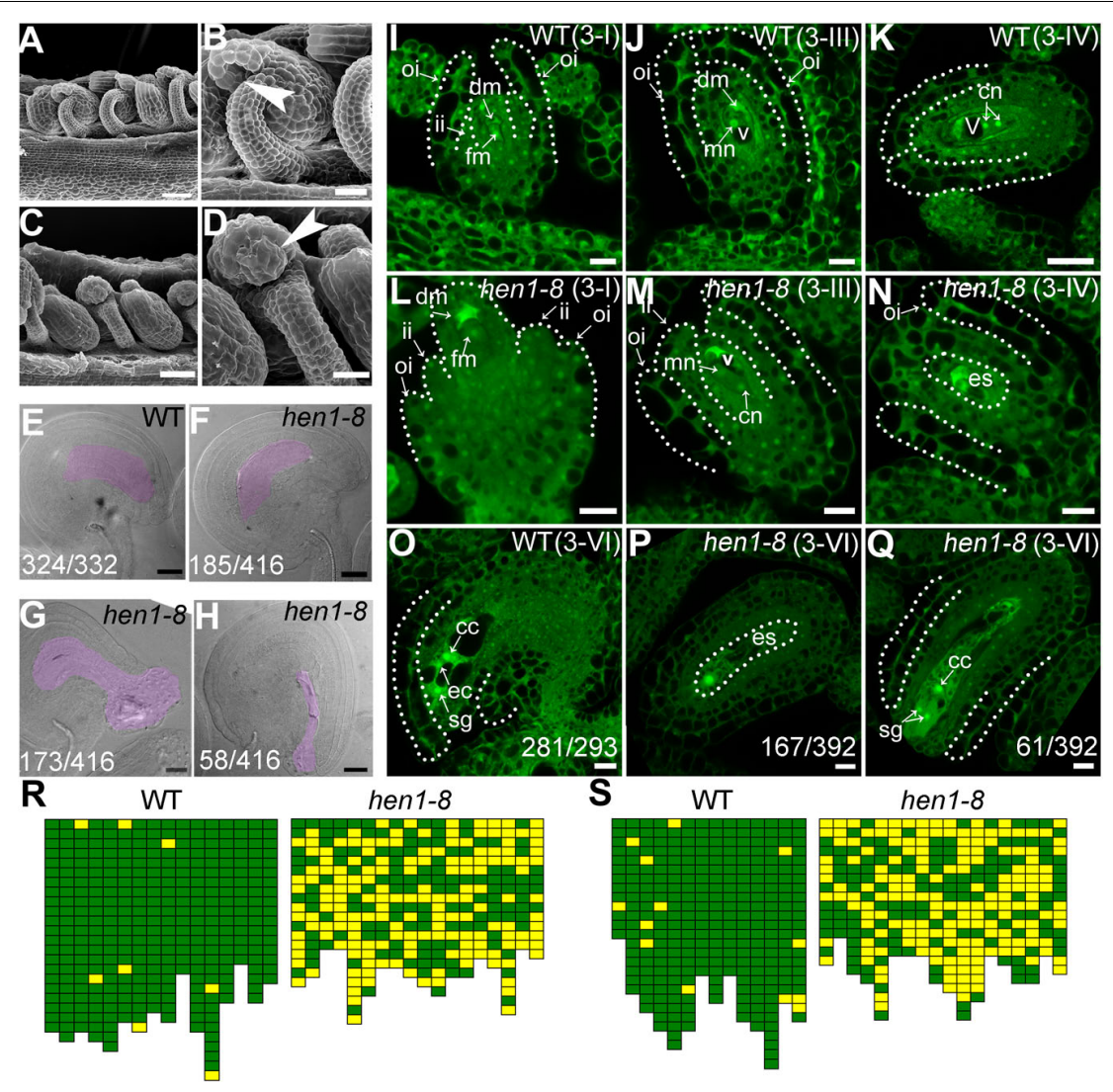

FIGURE 2 | hen1-8 is defective in ovule development. (A-D) Scanning electron micrographs (SEMs) of mature ovules from wild type (A,B) or from hen1-8 (C,D). (B) and $(\mathbf{D})$ are close-ups of $(\mathbf{A})$ and $(\mathbf{C})$, respectively. Arrows in (B,D) point at the micropyle. (E-H) Whole-mount ovule clearing of wild type (E) or hen1-8 (F-H). The ovule in (F) or in (G-H) represents normal or abnormal types, respectively. Embryo sacs are highlighted with lilac. (I-Q) Confocal laser scanning microscopy (CLSM) of wild-type (I-K,O) or hen1-8 (L-N,P,Q) ovules at stage 3-I (I,L), 3-III (J,M), 3-IV (K,N), or 3-VI (O-Q). Ovules were stained with PI and mid-optical sections are shown. For (I-Q), only hen1-8 ovules with visible nuclei were documented. For (E-H) and (O-Q), numbers on the bottom of each image indicate displayed ovules/total ovules examined. Cc, central cell; cn, chalazal nucleus; dm, degenerating megaspore; ec, egg cell; es, embryo sac; fm, functional megaspore; ii, inner integument; oi, outer integument; mn, micropylar nucleus; sg, synergid cell; v, vacuole. (R-S) Quantitative analysis of ovule development by ovule clearing (R) or by optical sections $\mathbf{( S )}$. Each pistil examined was represented by two neighboring columns; the number of cubes in each column indicates the number of countable ovules; normal and abnormal ovules (R) or embryo sacs (S) are displayed in green and yellow, respectively. Bars = $50 \mu \mathrm{m}$ for (A,C); $20 \mu \mathrm{m}$ for (B,D,E-H); $10 \mu \mathrm{m}$ for (I-J,L-Q); $20 \mu \mathrm{m}$ for (K).

hardly reaching the length of the inner integuments (Figure 2L). At this stage, functional megaspore (FM) was formed both in wild type and in hen 1-8 (Figures 2I,L). In wild type, from stage 3 -III to maturation, the outer integuments continued extended growth, finally enclosing the inner integuments (Figure 2J,K). Every mature ovules of wild type contains an embryo sac with a central cell, an egg cell and two synergid cells (Figure 20). By contrast, the outer integuments of hen 1-8 failed to enclose the inner structure (Figures 2M,N). At maturation, these ovules contain embryo sacs with abnormal cellular structures such that only one nucleus was visible (Figures $2 \mathbf{P}, \mathbf{Q}, \mathbf{S}$ ).

To provide further evidence that HEN1 is the causative gene for the observed ovule defects in hen1-8, we performed additional experiments. Because hen 1-2 is an allelic HEN1 mutant in Landsberg errecta (Ler) that contains exactly the same site mutation as in hen1-8 (Yu et al., 2010), we first examined ovule development of hen 1-2 by CLSM. Indeed, hen1-2 showed the same ovule defects as those of hen1-8 (Supplementary
Figure S3). Next, we crossed hen 1-8 and hen1-2 and examined ovules of the F1 progenies. Ovules of the F1 progenies from the cross showed exactly the same defects (Supplementary Figure S4). These results indicated defective outer integument growth affected embryo sac development when HEN1 is mutated.

\section{hen 1-8 Ovules Showed Reduced Pollen Tube Attraction}

CLSM of Pro ES1 $_{1}$ NLS-YFP;hen1-8 in which a nucleustargeted YFP was driven by an embryo sac-specific promoter (Pagnussat et al., 2009), often showed one nucleus, sometimes no nucleus at all, in the embryo sac in contrast to the eight nuclei structure in wild-type ovules (Supplementary Figure S5), suggesting that embryo sac development was compromised due to sporophytic defects in hen1-8. This result is also consistent with those obtained by optical section of mature hen 1-8 ovules (Figure 2). 
Because the embryo sac within an ovule attracts pollen tubes for fertilization (Higashiyama and Yang, 2017), abnormal embryo sacs due to defective integument growth might be the reason for reduced female fertility in hen1-8 (Figure 1). To test this hypothesis, wild-type or hen1-8 pistils were emasculated, and hand-pollinated with Pro $_{L A T 52}$ :GUS pollen and pollen tube attraction at $12 \mathrm{~h}$ after pollination (HAP) was examined by histochemical GUS staining. In contrast to wild type in which almost all ovules were targeted by a pollen tube, as indicated by a blue blob inside embryo sacs (Figures $3 \mathrm{~A}, \mathrm{C}$ ), over half of hen 1-8 ovules failed to attract a pollen tube (Figures 3B,D). By aniline blue staining of pistils at $48 \mathrm{HAP}$, we determined that most wild-type ovules were fertilized as indicated by size increase (Figure 3E). By contrast, around half of hen 1-8 ovules were not targeted by pollen tubes and were not fertilized (Figures 3F,G). Therefore, we concluded that defective embryo sac development in hen 1-8 resulted in its reduced female fertility.

\section{hyl1-2 Mimicked Ovule Defects of hen 1-8}

Ovule defects of hen 1-8 were likely due to compromised miRNA processing because HEN1 is critical for the processing of various miRNAs (Yu et al., 2005, 2010; Zhao et al., 2012) and sin1/dcl17 showed a similar phenotype (Robinson-Beers et al., 1992). To provide further evidence that the miRNA processing pathway was critical for ovule development, we also examined hyll2 (Vazquez et al., 2004), a null mutant of HYL1 whose severely reduced fertility was restored to the wild-type level by exogenous HYL1 (Lian et al., 2013). Female gametophytes of hyl1-2 transmitted comparably to those of wild type (Xiong et al., 2020), indicating that HYL1 is not required for the development of female gametophytes. However, the homozygous hyl1-2 showed a significantly reduced seed set due to sporophytic female defects (Figures 4A-E). By ovule whole-mount analysis (Figures 4F-G), optical sections (Figures 4H-I), and SEM analysis (Figures $\mathbf{4 L}-\mathbf{M}$ ), we demonstrated that hyl1-2 was defective in ovule development due to the growth arrest of outer integuments. Because of the defects, hyll-2 ovules showed a reduced ability to attract pollen tubes compared with those of wild type (Figures $4 \mathbf{J}-\mathbf{K}$ ), leading to significantly reduced female fertility (Figures 4B,D,E).

\section{HEN1 Functions in a Cell-Autonomous Way}

hen 1-8 shows vegetative growth retardation (Chen et al., 2002), which could have an impact in female fertility. To exclude the possibility that ovule developmental defect of hen1-8 was resulted from its reduced vegetative growth, we attempted to downregulate the expression of HEN1 specifically in outer integuments by using the outer integument-specific promoter

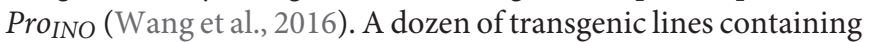
either Pro INO:amiR-HEN1 (artificial microRNA-HEN1) were generated. The transgenic plants were comparable to that of wild
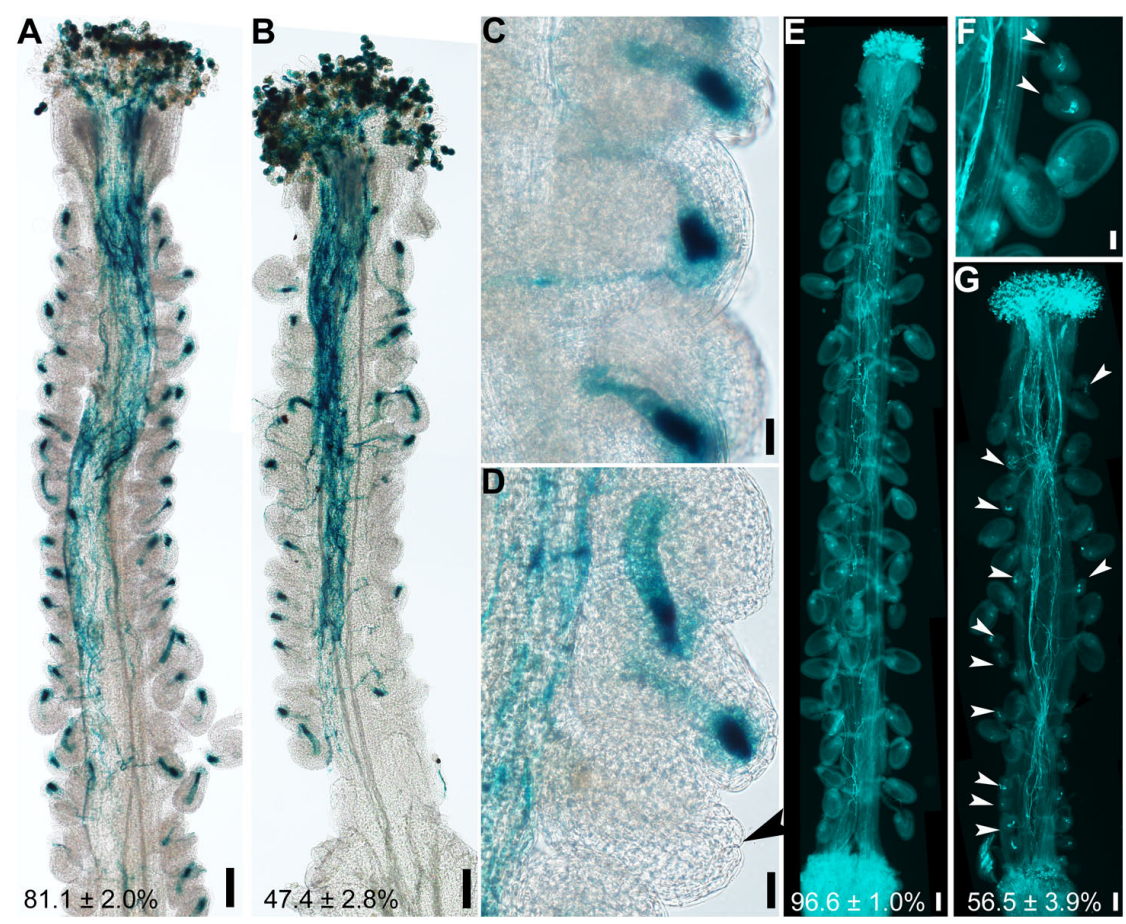

FIGURE 3 | hen 1-8 ovules are compromised in pollen tube guidance. (A-D) Histochemical GUS staining of wild-type (A,C) or hen 1-8 (B,D) pistils at 12 h after pollination (HAP) with PrO LAT 52 $_{2}$ :GUS pollen. Arrowheads point at an ovule that failed to attract pollen. (E-G) Aniline blue staining of wild-type (E) or hen 1-8 (F-G) pistils at $48 \mathrm{HAP}$ with wild-type pollen. Arrowheads point at ovules that did not develop as a result of fertilization failure. Two to three overlapping high-magnification images were taken for one pistil and overlaid with Photoshop (Adobe) to show the whole pistil (A,B,E,G). Numbers at the bottom of (A), (B), (C), (D) are quantification of targeted ovules out of total ovules. Results are means $\pm \mathrm{SD}(n=15)$. Bars $=100 \mu \mathrm{m}$ for $(\mathbf{A}, \mathbf{B}, \mathbf{E}, \mathbf{G}), 20 \mu \mathrm{m}$ for $(\mathbf{C}, \mathbf{D}, \mathbf{F})$. 


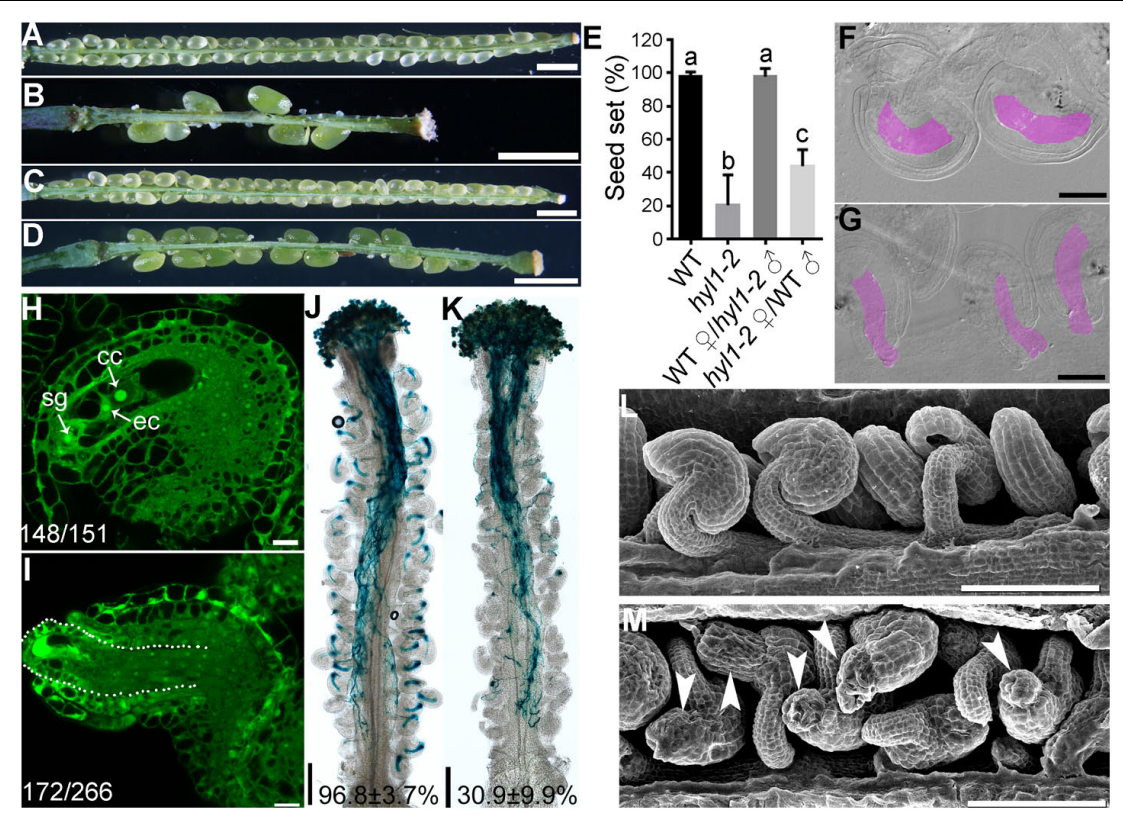

FIGURE 4 | hyl1-2 is defective in ovule development, similar to hen1-8. (A-D) Representative silique of wild type (A), hyl1-2 (B), wild type pollinated with hy/1-2 pollen (C), or hyl1-2 pollinated with wild-type pollen (D). (E) Quantitative analysis of seed set. Results are means \pm SD ( $n=15)$. Different numbers indicate significantly different groups (One-Way ANOVA, Tukey's multiple comparisons test, $P<0.05)$. (F,G) Whole-mount ovule clearing of wild type (F) or hyl1-2 (G). Embryo sacs are highlighted with lilac. $(\mathbf{H}, \mathbf{I})$ CLSM of wild-type $\mathbf{( H )}$ or hyl1-2 (I) ovules at stage 3-VI. Ovules were stained with PI and mid-optical sections are shown. Numbers on the bottom of each image indicate displayed ovules/total ovules examined. cc, central cell; ec, egg cell; sg, synergid cell. (J,K) Histochemical GUS staining of wild-type (J) or hyl1-2 (K) pistils at 12 HAP with PrOLAT52:GUS pollen. Two to three overlapping high-magnification images were taken for one pistil and overlaid with Photoshop (Adobe) to show the whole pistil. Numbers at the bottom are quantification of targeted ovules out of total ovules. Results are means \pm SD $(n=15)$. (L,M) SEMs of mature ovules from wild type (L) or from hyl1-2 (M). Arrowheads point at ovules with protruding embryo sac. Bars = 1 mm for $(\mathbf{A}-\mathbf{D}) ; 50$ m for $\mathbf{( F , G ) ; ~} 10 \mu \mathrm{m}$ for $\mathbf{( H - I ) ; ~} 200 \mu \mathrm{m}$ for $\mathbf{( J , K ) ; ~} 100 \mu \mathrm{m}$ for $\mathbf{( L , M ) . ~}$

type regarding the growth of vegetative tissues (Supplementary Figure S6), consistent with the use of outer-integument-specific promoter. However, seed set of the transgenic plants was compromised (Figures 5B,C,F). We examined two independent transgenic lines representing mildly or severely affected types by CLSM. In the line of Pro INO:amiR-HEN1 in which seed set was reduced by $50 \%$ (Figures $5 \mathbf{B}, \mathbf{F}$ ), half of the mature ovules showed abnormal number of nuclei in their embryo sacs while the integuments were morphologically indistinguishable from those of wild type (Figures $\mathbf{5 G}, \mathbf{H}$ ). In the line where there was hardly any seed set (Figures 5C,F), most mature ovules had no discernible outer integuments or nucleus structure in their embryo sacs (Figures 5I,J).

Because the specificity of Pro $_{I N O}$ used to downregulating HEN1 (Wang et al., 2016) and the constitutive expression of HEN1 in ovules (Supplementary Figure S2), we could not verify the downregulation of HEN1 in the Pro ${ }_{I N O}$ :amiR-HEN1 transgenic plants by quantitative real-time PCRs (qPCRs). Instead, we performed two experiments to support that HEN1 mediates integument growth in a cell-autonomous way. First, we generated Pro $_{35 s}$ :amiR-HEN1 transgenic plants and examined the transcript abundance of HEN1 in transgenic seedlings by qPCRs. In randomly selected two Pro 35 s:amiR-HEN1 independent lines, HEN1 abundance was significantly reduced compared to that in wild type (Supplementary Figure S7), suggesting that the amiR-HEN1 expression did reduce the mRNA level of HEN1. Consistently, the transgenic plants were shorter and smaller than wild-type plants (Supplementary Figure S7). Second, we used a RNA interference (RNAi) approach instead of amiR to downregulate HEN1 specifically in outer integuments. The Pro INO:HEN1-RNAi transgenic plants phenocopied Pro $_{I N O}$ :amiR-HEN1 in reduced seed set (Figures 5D,E,F) and defective ovule development (Figures $5 \mathrm{~K}-\mathbf{N}$ ). These results suggested that HEN1 mediates outer integument growth in a cell-autonomous way.

\section{Auxin Distribution but Not the Asymmetric PIN1 Localization Was Compromised in hen1-8 Ovules}

Auxin is a determinant factor in ovule development (Benkova et al., 2003; Bencivenga et al., 2012; Ceccato et al., 2013). Both auxin receptors and response factors are regulated by miRNAs whose processing depends on the DCL1-HEN1-HYL1 pathway (Navarro et al., 2006; Gutierrez et al., 2009; Ren et al., 2012; Zhao et al., 2012). Therefore, we examined auxin responses by introducing DR5:GFP (Ulmasov et al., 1997) into hen1-8 and examining GFP distribution. In wild type, GFP signals were detected only in the epidermal cell layer of the nucellus at stage 2-III when both outer and inner integuments were initiated (Figure 6A) and at stage 3-I when outer integuments underwent rapid growth to establish ovule anatrophy (Figure 6B). At 


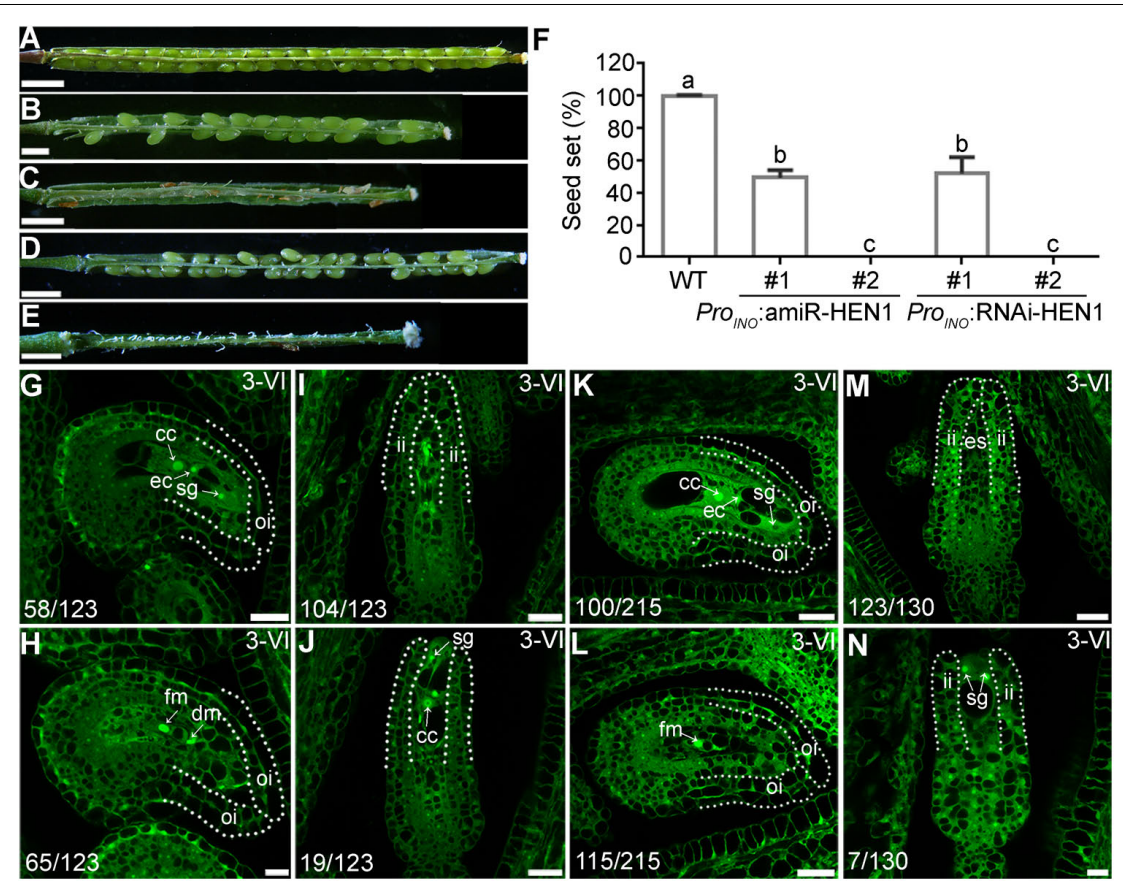

FIGURE 5 | Downregulating HEN1 in outer integuments mimicked hen1-8 in ovule development. (A-E) Representative silique from wild type (A), two lines of ProINO:amiR-HEN1 (B,C), and two lines of ProINO:HEN1-RNAi (D,E) transgenic plants. For some pistils, two to three overlapping high-magnification images were taken and overlaid with Photoshop (Adobe). (F) Quantification of seed set. Results are means \pm SD $(n=15)$. Different numbers indicate significantly different groups (One-Way ANOVA, Tukey's multiple comparisons test, $P<0.05)$. (G-N) CLSM of representative ovules at stage 3-VI from the \#1 (G,H) or \#2 (I,J) line of

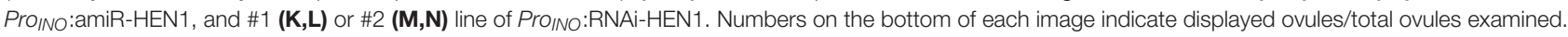
Dotted lines illustrate either outer integuments (oi) or inner integuments (ii). Cc, central cell; ec, egg cell; sg, synergid. Bars = $1 \mathrm{~mm}$ for (A,C,D); 500 $\mu \mathrm{m}$ for (B,E); $20 \mu \mathrm{m}$ for $(\mathbf{G}, \mathbf{I}, \mathbf{K}, \mathbf{M}, \mathbf{J}, \mathbf{L}) ; 10 \mu \mathrm{m}$ for $(\mathbf{H}, \mathbf{N})$.

maturation, GFP signals were hardly visible in ovules except in the vascular tissues of the funiculus (Figure 6C). The GFP distribution of hen 1-8 was similar to, albeit weaker than, that of wild type at early stages (Figure 6D) and at maturation in morphologically normal hen 1-8 ovules (Figure 6F). However, at stage $3-\mathrm{I}$, auxin maximum was expanded from the nucellus to the developing female gametophytes of hen 1-8 ovules (Figure 6E), suggesting a spatially disturbed auxin response.

Because PIN1 is the key auxin efflux carrier responsible for auxin distribution during ovule development (Ceccato et al., 2013), we also generated the PIN1:GFP; hen 1-8 plants to examine its distribution. As reported previously (Ceccato et al., 2013; Wang et al., 2016), PIN1 was asymmetrically distributed at the epidermal cells of the nucellus during ovule development (Figures 6G-H) and restricted to the funiculus at maturation (Figure 6I). No difference of PIN1 distribution was observed between wild type and hen1-8 (Figures 6J-L), indicating that compromised auxin maximum in hen1-8 ovules was likely resulted from signaling rather than auxin transport.

\section{Suppressing the Ectopic Expression of $A R F 6$ and $A R F 8$ by Introducing arf6;arf8 Did Not Rescue Ovule Defects of hen1-8}

Mutations of DCL1 (Robinson-Beers et al., 1992), HEN1, and HYL1 resulted in defective ovule development, suggesting a role of miRNAs in this process. Among miRNAs whose accumulation relies on HEN1 (Ren et al., 2012; Zhao et al., 2012), miRNA167 was demonstrated a positive regulator for ovule development by suppressing the expression of ARF6 and ARF8 (Wu et al., 2006; Yao et al., 2019). Indeed, ovule development of the mir167 mutants was largely recovered by introducing either arf6 or arf8 (Yao et al., 2019).

To test the possibility that ectopic expression of ARF6 and ARF8 resulted in the arrest of integuments in hen1-8, we performed the following experiments. First, we examined the expression of ARF6 and ARF8 in hen 1-8 ovules by RNA in situ hybridization. As reported previously (Wu et al., 2006), ARF6 (Figures 7A,B) and ARF8 (Figures 7E-F) were highly expressed in the funiculus during ovule development in wild type. By contrast, signals of either ARF6 (Figures 7C,D) or ARF8 (Figures 7G,H) were detected in whole ovules of hen 1-8, indicating its ectopic expression. Second, by quantitative RTPCR (qRT-PCR), we could verify that transcript abundance of both ARF6 and ARF8 was significantly increased in hen18 ovules (Figures $7 \mathbf{I}, \mathbf{J}$ ). Third, we introduced the mutants of ARF6 and ARF8, i.e., arf6-1 and arf8-3 respectively, into hen1-8 and analyzed the resultant hierarchy mutants. Introducing arf6-1 or arf8-3 alone into hen1-8 did not affect vegetative growth whereas the arf6-1;arf8-3; hen1-8 showed a severe growth retardation (Supplementary Figure S8). Close examination of mature ovules from different genotypes showed 


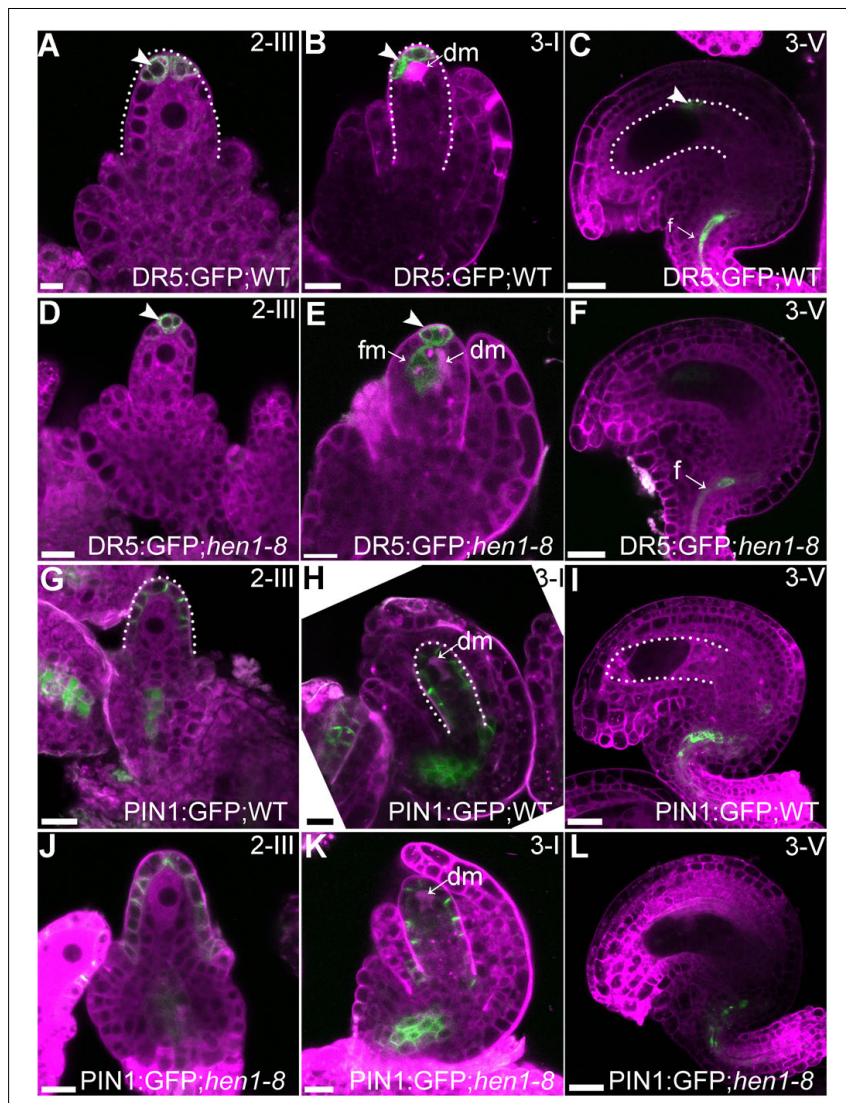

FIGURE 6 | Auxin responses but not the asymmetric PIN1 localization were compromised in hen 1-8 during ovule development. (A-F) DR5:GFP in wild-type (A-C) or in hen1-8 (D-F) ovules at different stages by CLSM Arrowheads indicate DR5 signals. (G-L) PIN1:GFP in wild-type (G-I) or in hen 1-8 (J-L) ovules at different stages by CLSM. Ovules were stained with lysotracker red (magenta). dm: degenerating megaspore. Bars $=10 \mu \mathrm{m}$.

that either arf6-1, or arf8-3, or the arf6-1;arf8-3 double mutant could not restore ovule developmental defects of hen1-8 (Supplementary Figure S9), suggesting that ectopic expression of ARF6 and ARF8 was not the reason for the arrest of hen 1-8 integuments.

\section{DISCUSSION}

We demonstrate here that mutations of HEN1 and HYL1 resulted in the arrest of integument growth during ovule development, similar to those of DCL1. Because female gametophyte of hen 1-8 (Supplementary Table S2) and hyl1-2 (Xiong et al., 2020) transmits comparably that of wild type, the embryo sac defects observed in hen1-8 and hyl1-2, possibly also in sin1/dcl1-7 (Robinson-Beers et al., 1992), were resulted from sporophytic defects through intercellular signaling (Bencivenga et al., 2011), as being reported (Wang et al., 2016; Liu et al., 2019). Embryo sacs send signals to guide directional pollen tube growth (Higashiyama and Yang, 2017). It is therefore understandably that hen1-8 or hyl1-2 ovules showed a significantly reduced ability to attract pollen tubes (Figures 3, 4). hen1-8 is a hypomorphic allele ( $\mathrm{Yu}$ et al., 2010) whereas hyll-2 is a null allele (Han et al., 2004; Kurihara et al., 2006; Dong et al., 2008), which explains the different severity of ovule defects (Figures 1, 4).

A recent study reported that the mutations of $H Y L 1, D C L 1$, or HEN1 caused a reduced number of pollen and megaspore mother cells (Oliver et al., 2017). Our results strongly suggested that fertility reduction in hen1-8 was due to abnormal ovule development (Figure 3). First, hen 1-8 as the pollen donor to wild type resulted in a full seed set (Figure 1), indicating normal pollen function. Second, pollen development is comparable between hen1-8 and wild type, despite the relatively small size of hen1-8 anthers (Supplementary Figure S1). Third, the heterozygous hen 1-8 mutant also produced full seed set (Figure 1), arguing against female gametophytic defects. Indeed, reciprocal crosses between wild type and the heterozygous hen18 indicated that both male and female transmission of hen 1-8 are normal (Supplementary Table S1). The discrepancy between the previous report (Oliver et al., 2017) and ours might be due to the fact that hen 1-8 and hen1-2 are weaker alleles of HEN1.

Despite that the hen1-8 plants showed sub-optical vegetative growth (Chen et al., 2002; Ren et al., 2012), we believe that HEN1 works in a cell-autonomous way to regulate the asymmetric growth of outer integuments. Downregulating HEN1 specifically in outer integuments was sufficient to cause ovule defect similar to, even more severe than, that of hen 1-8 (Figure 5), without affecting vegetative growth (Supplementary Figure S6). Because the cell-specific feature of these transgenic lines, it is difficult, if possible, to examine the reduction of HEN1 transcript abundance. To make it additionally difficult, in the more severely affected RNAi or amiR lines, the growth of outer integuments was arrested very early on. But the two different constructs used to downregulating HEN1 in outer integuments gave the same results, strongly supporting a cell autonomous role of HEN1 in outer integuments.

The accumulation of miRNA167 was significantly reduced in hen1 mutants (Yu et al., 2010; Ren et al., 2012). Consistently, ARF6 and ARF8, major targets of miRNA167 (Nagpal et al., 2005; Wu et al., 2006; Yao et al., 2019; Zheng et al., 2019), were ectopically expressed in hen1-8 (Figure 7). However, introducing arf6 or arf8 did not suppress developmental defects of hen1-8 ovules (Supplementary Figure S9). The inability is unlikely to have caused by substantially compromised growth of the arf61; arf8-3; hen1-8 triple mutant since introducing either arf6-1 or arf8-3 didn't aggravate the growth of hen 1-8 but yet was not able to rescue its defects (Supplementary Figure S8). A more likely possibility is that more miRNAs downstream of HEN1 play roles in this process. Auxin maximum was altered in developing ovules of hen1-8 such that DR5 signals were expanded to the developing female gametophytes in hen 1-8 rather than restricted to the nucellus as in wild type (Figure 6). Because the sporophytic integuments affect FG development (Bencivenga et al., 2011; Wang et al., 2016; Liu et al., 2019), these results indicated that auxin signaling in integuments was compromised in hen18. Indeed, genes encoding auxin receptors are also targets of 


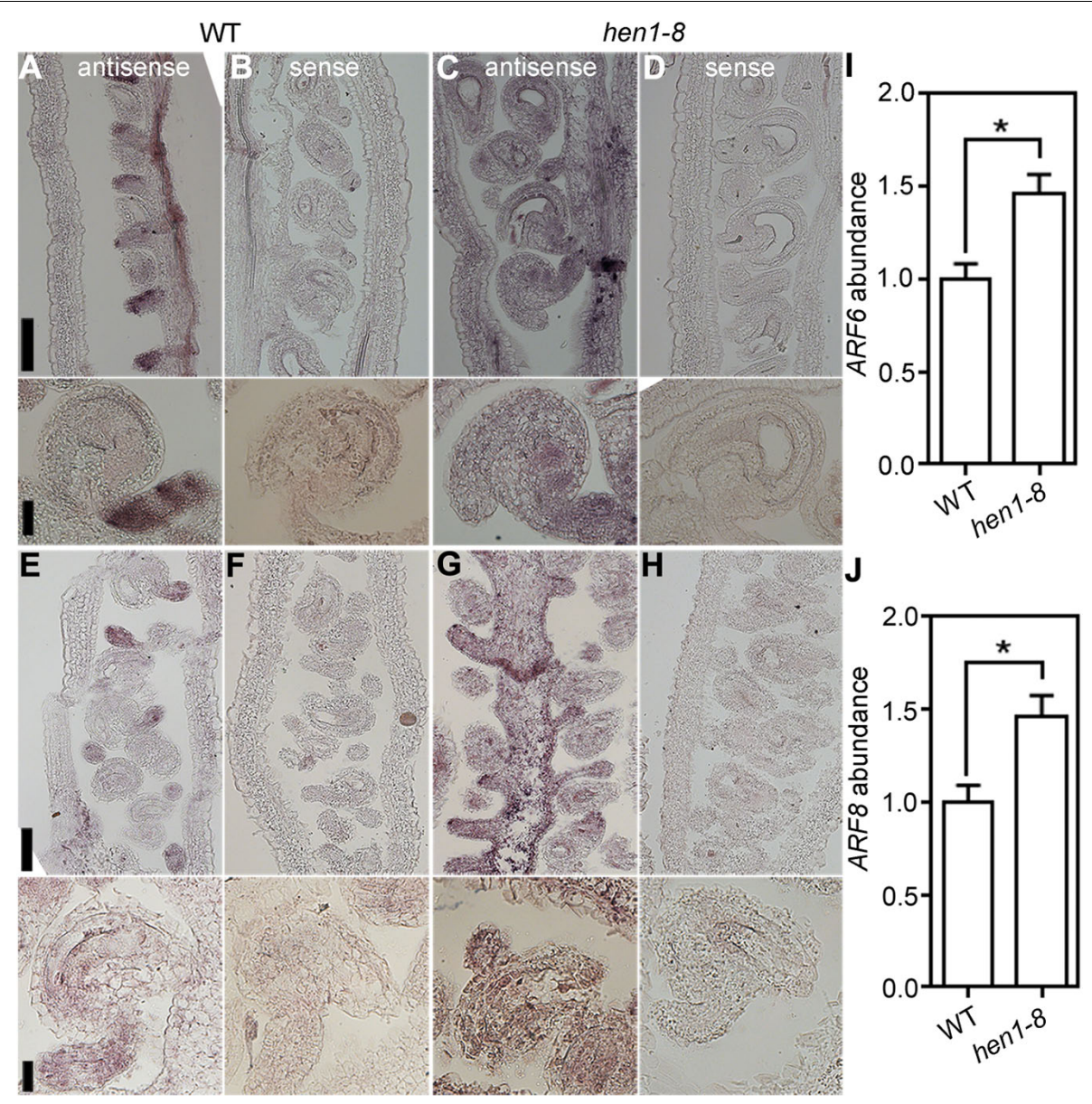

FIGURE 7 | ARF6 and ARF8 are ectopically expressed in hen1-8 ovules. (A-D) Stage 3-IV wild-type (A,B) or hen1-8 (C,D) ovules labeled by the ARF6 antisense probe $\mathbf{( A , C )}$ or sense probe (B,D). (E-H) Stage 3-IV wild-type $\mathbf{( E , F ) ~ o r ~ h e n 1 - 8 ~ ( G , H ) ~ o v u l e ~ l a b e l e d ~ b y ~ t h e ~ A R F 8 ~ a n t i s e n s e ~ p r o b e ~}(\mathbf{E}, \mathbf{G})$ or sense probe $(\mathbf{F}, \mathbf{H})$. Funiculus of wild-type ovules whereas integuments of hen1-8 ovules show strong signals by the antisense probes. (I,J) Relative ARF6 (I) or ARF8 (J) abundance in mature ovules of wild type or hen 1-8 by qRT-PCRs. GAPDH and TUBULIN2 were used as internal controls. Results shown are means \pm SE $(n=3)$. Asterisks indicate significant difference ( $t$-test, $P<0.01$ ). Bars $=50 \mu \mathrm{m}$ for whole views of pistils and $20 \mu \mathrm{m}$ for close-ups of ovules.

miRNAs (Navarro et al., 2006; Gutierrez et al., 2009). A genomewide small RNA sequencing will be useful to identify miRNAs that are expressed in integuments and whose reduced levels result in the arrest of integument growth in mutants of the DCL1-HEN1-HYL1 pathway.

\section{DATA AVAILABILITY STATEMENT}

All datasets generated for this study are included in the article/Supplementary Material.

\section{AUTHOR CONTRIBUTIONS}

S-JW and SC performed all the experiments with the assistance of R-MZ and C-YD. SL and YZ conceived and supervised the project and secured the funding. S-JW, SL, and YZ analyzed the data. YZ wrote the article with input from all authors.

\section{FUNDING}

This work was supported by Natural Science Foundation of China (31871422 and 31771558 to SL, 31970332 and 31625003 to YZ). YZ's laboratory is partially supported by Tai-Shan Scholar Program by Shandong Provincial Government.

\section{ACKNOWLEDGMENTS}

We thank Prof. Xian Sheng Zhang for DR5:GFP and PIN1:GFP; Prof. Guo-Dong Ren for the kind gift of hen1-8 and hen1-2; Prof. Yijun Qi for hyl1-2.

\section{SUPPLEMENTARY MATERIAL}

The Supplementary Material for this article can be found online at: https://www.frontiersin.org/articles/10.3389/fpls.2020.00397/ full\#supplementary-material 


\section{REFERENCES}

Baranauske, S., Mickute, M., Plotnikova, A., Finke, A., Venclovas, C., Klimasauskas, S., et al. (2015). Functional mapping of the plant small RNA methyltransferase: HEN1 physically interacts with HYL1 and DICER-LIKE 1 proteins. Nucleic Acids Res. 43, 2802-2812. doi: 10.1093/nar/gkv102

Bencivenga, S., Colombo, L., and Masiero, S. (2011). Cross talk between the sporophyte and the megagametophyte during ovule development. Sex Plant Reprod. 24, 113-121. doi: 10.1007/s00497-011-0162-3

Bencivenga, S., Simonini, S., Benkova, E., and Colombo, L. (2012). The transcription factors BEL1 and SPL are required for cytokinin and auxin signaling during ovule development in Arabidopsis. Plant Cell 24, 2886-2897. doi: $10.1105 /$ tpc.112.100164

Benkova, E., Michniewicz, M., Sauer, M., Teichmann, T., Seifertova, D., Jurgens, G., et al. (2003). Local, efflux-dependent auxin gradients as a common module for plant organ formation. Cell 115, 591-602. doi: 10.1016/s0092-8674(03)00924-3

Ceccato, L., Masiero, S., Sinha Roy, D., Bencivenga, S., Roig-Villanova, I., Ditengou, F. A., et al. (2013). Maternal control of PIN1 is required for female gametophyte development in Arabidopsis. PLoS One 8:e66148. doi: 10.1371/journal.pone. 0066148

Chen, X., Liu, J., Cheng, Y., and Jia, D. (2002). HEN1 functions pleiotropically in Arabidopsis development and acts in C function in the flower. Development 129, 1085-1094.

Chevalier, E., Loubert-Hudon, A., Zimmerman, E. L., and Matton, D. P. (2011). Cell-cell communication and signalling pathways within the ovule: from its inception to fertilization. New Phytol. 192, 13-28. doi: 10.1111/j.1469-8137. 2011.03836.x

Christensen, C. A., King, E. J., Jordan, J. R., and Drews, G. N. (1997). Megagametogenesis in Arabidopsis wild type and the Gf mutant. Sex Plant Reprod. 10, 49-64. doi: 10.1007/s004970050067

Colombo, L., Battaglia, R., and Kater, M. M. (2008). Arabidopsis ovule development and its evolutionary conservation. Trends Plant Sci. 13, 444-450. doi: 10.1016/ j.tplants.2008.04.011

Curtis, M. D., and Grossniklaus, U. (2003). A gateway cloning vector set for highthroughput functional analysis of genes in planta. Plant Physiol. 133, 462-469. doi: $10.1104 /$ pp. 103.027979

Dong, Z., Han, M. H., and Fedoroff, N. (2008). The RNA-binding proteins HYL1 and SE promote accurate in vitro processing of pri-miRNA by DCL1. Proc. Natl. Acad. Sci. U.S.A. 105, 9970-9975. doi: 10.1073/pnas.0803356105

Drews, G. N., Lee, D., and Christensen, C. A. (1998). Genetic analysis of female gametophyte development and function. Plant Cell 10, 5-17. doi: 10.1105/tpc. 10.1.5

Drews, G. N., and Yadegari, R. (2002). Development and function of the angiosperm female gametophyte. Annu. Rev. Genet. 36, 99-124. doi: 10.1146/ annurev.genet.36.040102.131941

Elliott, R. C., Betzner, A. S., Huttner, E., Oakes, M. P., Tucker, W. Q., Gerentes, D., et al. (1996). AINTEGUMENTA, an APETALA2-like gene of Arabidopsis with pleiotropic roles in ovule development and floral organ growth. Plant Cell 8 , 155-168. doi: 10.1105/tpc.8.2.155

Gross-Hardt, R., Lenhard, M., and Laux, T. (2002). WUSCHEL signaling functions in interregional communication during Arabidopsis ovule development. Genes Dev. 16, 1129-1138. doi: 10.1101/gad.225202

Guo, J., Wang, F., Song, J., Sun, W., and Zhang, X. S. (2010). The expression of Orysa;CycB1;1 is essential for endosperm formation and causes embryo enlargement in rice. Planta 231, 293-303. doi: 10.1007/s00425-009-1051-y

Gutierrez, L., Bussell, J. D., Pacurar, D. I., Schwambach, J., Pacurar, M., and Bellini, C. (2009). Phenotypic plasticity of adventitious rooting in Arabidopsis is controlled by complex regulation of AUXIN RESPONSE FACTOR transcripts and microRNA abundance. Plant Cell 21, 3119-3132. doi: 10.1105/tpc.108. 064758

Han, M. H., Goud, S., Song, L., and Fedoroff, N. (2004). The Arabidopsis doublestranded RNA-binding protein HYL1 plays a role in microRNA-mediated gene regulation. Proc. Natl. Acad. Sci. U.S.A. 101, 1093-1098. doi: 10.1073/pnas. 0307969100

Higashiyama, T., and Yang, W. C. (2017). Gametophytic pollen tube guidance: attractant peptides, gametic controls, and receptors. Plant Physiol. 173, 112 121. doi: $10.1104 /$ pp.16.01571
Klucher, K. M., Chow, H., Reiser, L., and Fischer, R. L. (1996). The AINTEGUMENTA gene of Arabidopsis required for ovule and female gametophyte development is related to the floral homeotic gene APETALA2. Plant Cell 8, 137-153. doi: 10.1105/tpc.8.2.137

Kurihara, Y., Takashi, Y., and Watanabe, Y. (2006). The interaction between DCL1 and HYL1 is important for efficient and precise processing of pri-miRNA in plant microRNA biogenesis. RNA 12, 206-212. doi: 10.1261/rna.2146906

Kurihara, Y., and Watanabe, Y. (2004). Arabidopsis micro-RNA biogenesis through Dicer-like 1 protein functions. Proc. Natl. Acad. Sci. U.S.A. 101, 12753-12758. doi: 10.1073/pnas.0403115101

Li, S., Ge, F. R., Xu, M., Zhao, X. Y., Huang, G. Q., Zhou, L. Z., et al. (2013). Arabidopsis COBRA-LIKE 10, a GPI-anchored protein, mediates directional growth of pollen tubes. Plant J. 74, 486-497. doi: 10.1111/tpj.12139

Lian, H., Li, X., Liu, Z., and He, Y. (2013). HYL1 is required for establishment of stamen architecture with four microsporangia in Arabidopsis. J. Exp. Bot. 64, 3397-3410. doi: 10.1093/jxb/ert178

Lieber, D., Lora, J., Schrempp, S., Lenhard, M., and Laux, T. (2011). Arabidopsis WIH1 and WIH2 genes act in the transition from somatic to reproductive cell fate. Curr. Biol. 21, 1009-1017. doi: 10.1016/j.cub.2011.05.015

Liu, H. H., Xiong, F., Duan, C. Y., Wu, Y. N., Zhang, Y., and Li, S. (2019). Importin $\beta 4$ mediates nuclear import of GRF-interacting factors to control ovule development in Arabidopsis. Plant Physiol. 179, 1080-1092. doi: 10.1104/ pp.18.01135

Lu, C., and Fedoroff, N. (2000). A mutation in the Arabidopsis HYL1 gene encoding a dsRNA binding protein affects responses to abscisic acid, auxin, and cytokinin. Plant Cell 12, 2351-2366.

McAbee, J. M., Hill, T. A., Skinner, D. J., Izhaki, A., Hauser, B. A., Meister, R. J., et al. (2006). ABERRANT TESTA SHAPE encodes a KANADI family member, linking polarity determination to separation and growth of Arabidopsis ovule integuments. Plant J. 46, 522-531. doi: 10.1111/j.1365-313X.2006.02717.x

Nagpal, P., Ellis, C. M., Weber, H., Ploense, S. E., Barkawi, L. S., Guilfoyle, T. J., et al. (2005). Auxin response factors ARF6 and ARF8 promote jasmonic acid production and flower maturation. Development 132, 4107-4118. doi: 10.1242/ dev.01955

Navarro, L., Dunoyer, P., Jay, F., Arnold, B., Dharmasiri, N., Estelle, M., et al. (2006). A plant miRNA contributes to antibacterial resistance by repressing auxin signaling. Science 312, 436-439. doi: 10.1126/science.1126088

Okushima, Y., Overvoorde, P. J., Arima, K., Alonso, J. M., Chan, A., Chang, C., et al. (2005). Functional genomic analysis of the AUXIN RESPONSE FACTOR gene family members in Arabidopsis thaliana: unique and overlapping functions of ARF7 and ARF19. Plant Cell 17, 444-463. doi: 10.1105/tpc.104.028316

Oliver, C., Pradillo, M., Jover-Gil, S., Cunado, N., Ponce, M. R., and Santos, J. L. (2017). Loss of function of Arabidopsis microRNA-machinery genes impairs fertility, and has effects on homologous recombination and meiotic chromatin dynamics. Sci. Rep. 7:9280. doi: 10.1038/s41598-017-07702-x

Olmedo-Monfil, V., Duran-Figueroa, N., Arteaga-Vazquez, M., Demesa-Arevalo, E., Autran, D., Grimanelli, D., et al. (2010). Control of female gamete formation by a small RNA pathway in Arabidopsis. Nature 464, 628-632. doi: 10.1038/ nature 08828

Pagnussat, G. C., Alandete-Saez, M., Bowman, J. L., and Sundaresan, V. (2009). Auxin-dependent patterning and gamete specification in the Arabidopsis female gametophyte. Science 324, 1684-1689. doi: 10.1126/science.1167324

Reiser, L., Modrusan, Z., Margossian, L., Samach, A., Ohad, N., Haughn, G. W., et al. (1995). The BELL1 gene encodes a homeodomain protein involved in pattern formation in the Arabidopsis ovule primordium. Cell 83, 735-742. doi: 10.1016/0092-8674(95)90186-8

Ren, G., Chen, X., and Yu, B. (2012). Uridylation of miRNAs by HEN1 SUPPRESSOR1 in Arabidopsis. Curr. Biol. 22, 695-700. doi: 10.1016/j.cub.2012. 02.052

Robinson-Beers, K., Pruitt, R. E., and Gasser, C. S. (1992). Ovule development in wild-type Arabidopsis and two female-sterile mutants. Plant Cell 4, 1237-1249. doi: $10.1105 /$ tpc.4.10.1237

Rogers, K., and Chen, X. (2013). Biogenesis, turnover, and mode of action of plant microRNAs. Plant Cell 25, 2383-2399. doi: 10.1105/tpc.113.113159

Schauer, S. E., Jacobsen, S. E., Meinke, D. W., and Ray, A. (2002). DICER-LIKE1: blind men and elephants in Arabidopsis development. Trends Plant Sci. 7, 487-491. doi: 10.1016/s1360-1385(02)02355-5 
Schneitz, K., Hulskamp, M., Kopczak, S. D., and Pruitt, R. E. (1997). Dissection of sexual organ ontogenesis: a genetic analysis of ovule development in Arabidopsis thaliana. Development 124, 1367-1376.

Schneitz, K., Hülskamp, M., and Pruitt, R. E. (1995). Wild-type ovule development in Arabidopsis thaliana: a light microscope study of cleared whole-mount tissue. Plant J. 7, 731-749. doi: 10.1046/j.1365-313X.1995.07050731.x

Shi, D. Q., and Yang, W. C. (2011). Ovule development in Arabidopsis: progress and challenge. Curr. Opin. Plant Biol. 14, 74-80. doi: 10.1016/j.pbi.2010.09.001

Sieber, P., Gheyselinck, J., Gross-Hardt, R., Laux, T., Grossniklaus, U., and Schneitz, K. (2004). Pattern formation during early ovule development in Arabidopsis thaliana. Dev. Biol. 273, 321-334. doi: 10.1016/j.ydbio.2004.05.037

Ulmasov, T., Murfett, J., Hagen, G., and Guilfoyle, T. J. (1997). Aux/IAA proteins repress expression of reporter genes containing natural and highly active synthetic auxin response elements. Plant Cell 9, 1963-1971. doi: 10.1105/tpc. 9.11.1963

Vazquez, F., Gasciolli, V., Crete, P., and Vaucheret, H. (2004). The nuclear dsRNA binding protein HYL1 is required for microRNA accumulation and plant development, but not posttranscriptional transgene silencing. Curr. Biol. 14, 346-351. doi: 10.1016/j.cub.2004.01.035

Villanueva, J. M., Broadhvest, J., Hauser, B. A., Meister, R. J., Schneitz, K., and Gasser, C. S. (1999). INNER NO OUTER regulates abaxial- adaxial patterning in Arabidopsis ovules. Genes Dev. 13, 3160-3169. doi: 10.1101/gad.13.23. 3160

Wang, J. G., Feng, C., Liu, H. H., Ge, F. R., Li, S., Li, H. J., et al. (2016). HAPLESS13mediated trafficking of STRUBBELIG is critical for ovule development in Arabidopsis. PLoS Genet. 12:e1006269. doi: 10.1371/journal.pgen.1006269

Wu, M. F., Tian, Q., and Reed, J. W. (2006). Arabidopsis microRNA167 controls patterns of ARF6 and ARF8 expression, and regulates both female and male reproduction. Development 133, 4211-4218. doi: 10.1242/dev.02602

Xiong, F., Duan, C. Y., Liu, H. H., Wu, J. H., Zhang, Z. H., Li, S., et al. (2020). Arabidopsis KETCH1 is critical for the nuclear accumulation of ribosomal proteins and gametogenesis. Plant Cell (in press). doi: 10.1105/tpc.19.00791
Yang, S. W., Chen, H. Y., Yang, J., Machida, S., Chua, N. H., and Yuan, Y. A. (2010). Structure of Arabidopsis HYPONASTIC LEAVES1 and its molecular implications for miRNA processing. Structure 18, 594-605. doi: 10.1016/j.str. 2010.02.006

Yao, X., Chen, J., Zhou, J., Yu, H., Ge, C., Zhang, M., et al. (2019). An essential role for miRNA167 in maternal control of embryonic and seed development. Plant Physiol. 180, 453-464. doi: 10.1104/pp.19.00127

Yu, B., Bi, L., Zhai, J., Agarwal, M., Li, S., Wu, Q., et al. (2010). siRNAs compete with miRNAs for methylation by HEN1 in Arabidopsis. Nucleic Acids Res. 38, 5844-5850. doi: 10.1093/nar/gkq348

Yu, B., Yang, Z., Li, J., Minakhina, S., Yang, M., Padgett, R. W., et al. (2005). Methylation as a crucial step in plant microRNA biogenesis. Science 307, 932-935. doi: 10.1126/science.1107130

Zhao, Y., Mo, B., and Chen, X. (2012). Mechanisms that impact microRNA stability in plants. RNA Biol. 9, 1218-1223. doi: 10.4161/rna.22034

Zheng, L., Nagpal, P., Villarino, G., Trinidad, B., Bird, L., Huang, Y., et al. (2019). miR167 limits anther growth to potentiate anther dehiscence. Development 146:dev174375. doi: 10.1242/dev.174375

Zhou, L. Z., Li, S., Feng, Q. N., Zhang, Y. L., Zhao, X., Zeng, Y. L., et al. (2013). PROTEIN S-ACYL transferase10 is critical for development and salt tolerance in Arabidopsis. Plant Cell 25, 1093-1107. doi: 10.1105/tpc.112.108829

Conflict of Interest: The authors declare that the research was conducted in the absence of any commercial or financial relationships that could be construed as a potential conflict of interest.

Copyright (c) $2020 \mathrm{Wei}$, Chai, Zhu, Duan, Zhang and Li. This is an open-access article distributed under the terms of the Creative Commons Attribution License (CC BY). The use, distribution or reproduction in other forums is permitted, provided the original author(s) and the copyright owner(s) are credited and that the original publication in this journal is cited, in accordance with accepted academic practice. No use, distribution or reproduction is permitted which does not comply with these terms. 